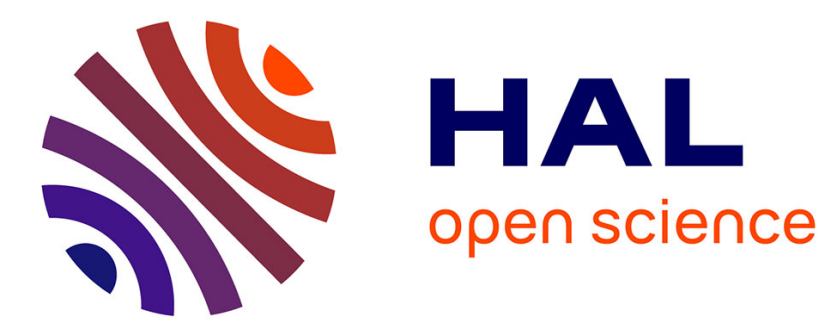

\title{
Modeling photon pair generation by second-order surface nonlinearity in silica nanofibers
}

\author{
Abderrahim Azzoune, Philippe Delaye, Gilles Pauliat
}

\section{To cite this version:}

Abderrahim Azzoune, Philippe Delaye, Gilles Pauliat. Modeling photon pair generation by secondorder surface nonlinearity in silica nanofibers. Journal of the Optical Society of America B, 2021, 38, pp.1057-1068. 10.1364/josab.418661 . hal-03279754

\section{HAL Id: hal-03279754 \\ https://hal.science/hal-03279754}

Submitted on 13 Jul 2021

HAL is a multi-disciplinary open access archive for the deposit and dissemination of scientific research documents, whether they are published or not. The documents may come from teaching and research institutions in France or abroad, or from public or private research centers.
L'archive ouverte pluridisciplinaire HAL, est destinée au dépôt et à la diffusion de documents scientifiques de niveau recherche, publiés ou non, émanant des établissements d'enseignement et de recherche français ou étrangers, des laboratoires publics ou privés. 


\title{
Modelling photon pair generation by second- order surface nonlinearity in silica nanofibers
}

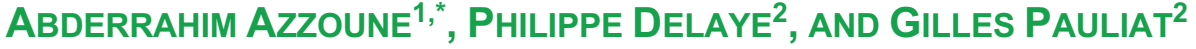 \\ 1) École Militaire Polytechnique, Laboratoire Systèmes Lasers, BP17, 16111, Bordj El Bahri, Algérie \\ 2) Université Paris-Saclay, Institut d'Optique Graduate School, CNRS, Laboratoire Charles Fabry, \\ 91127, Palaiseau, France \\ *azzoune.abderrahim@gmail.com
}

\begin{abstract}
In this paper, we present a design of an all-fiber source of correlated photon pairs based on standard telecommunications tapered fibers. We examine the generation of correlated photon pairs using parametric process $\chi^{(2)}$ in silica tapered optical fibers. This nonlinear process is ensured thanks to surface dipole and bulk multipole nonlinearities. The process of photons creation is modeled by taking into account the vector aspect of the propagation of the optical field in a silica nanofiber. The phase matching is provided by propagating the pump field in one spatial mode, while generating a photon pair in another spatial mode. The generation efficiency of photon pairs depends on diameter uniformity of the nanofiber after the manufacturing process. We size this nanofiber for a good optimization of photon pair generation efficiency, we report that the tolerance in diameter uniformity is $\Delta d=2 \mathrm{~nm}$ for a generation rate of photon pairs estimated to $N_{p h} \approx 22000$ pairs/s, for $1 \mathrm{~W}$ power pump and a nanofiber length of $1.1 \mathrm{~mm}$. Deposits on the nanofiber can be used in order to relax the manufacturing constraints on diameter to maximize the generation rate of photon pairs. As an example, the use of Polytetrafluoroethylene (PTFE) on the nanofiber applied as a cladding whose thickness is infinite makes it possible to relax the constraints on the nanofiber diameter. For the same $\Delta d=2 \mathrm{~nm}$, a generation rate of photon pairs estimated to $N_{p h} \approx 78000$ pairs $/ \mathrm{s}$ for $1 \mathrm{~W}$ power pump and a nanofiber length of $2.4 \mathrm{~mm}$ is predicted.
\end{abstract}

(C) 2021 Optical Society of America

\section{Introduction}

Optical fibers are a key component for quantum communications systems as a privileged media for qubit transportation due to their very low loss for propagating photons. They are also easily connected to other devices used to generate, process and detect these photons [1-5]. Nevertheless, this interfacing is not perfect and the study of fibered components to realize sources of photon pairs or quantum processing devices is a very active research field. The basis of fibered source of photon pairs is Spontaneous Four Wave Mixing (FWM) [6-11], that allows good performances but also presents some limitations. The main limitation is related to the Spontaneous Raman Scattering that creates uncorrelated noise photons in the vicinity of the emitted photons that are usually rather close to the pump [5]. Moreover, the intensity dependence of the FWM mechanism, favors the pulse regime and fibers are less often operated in the $\mathrm{CW}$ regime [12]. Both limitations could be raised by using a second-order nonlinearity to generate the pairs of photons through Spontaneous Parametric Down Conversion (SPDC), a solution widely used in nonlinear crystals or waveguides $[13,14]$ the most popular being Periodically Poled Lithium Niobate (PPLN) $[15,16]$. The problem is that silica is a centrosymmetric material that does not present naturally such a second-order nonlinearity in its bulk state. This problem had been solved using periodical poling of silica fibers $[17,18]$ but that solution requires complex process to make the fiber nonlinear and to achieve phase matching and may encounter some stability problem over time.

In this paper, we propose a new solution to realize SPDC in fibers using a tapered standard telecommunication fiber [19-23]. The second-order nonlinearity is established through surface 
dipole and bulk multipole nonlinearities, that are exalted due to the sub-wavelength diameter of the nanofiber. We first model the second-order nonlinear surface susceptibility at the surface of the silica nanofiber by taking into account the vector aspect of the propagation of the optical field. In a second step, we define modal phase matchings that are necessary to obtain SPDC. We size this nanofiber for a best optimization of photon pair generation efficiency. As an example, we propose to use Polytetrafluoroethylene (PTFE) as a deposit on the nanofiber in order to relax manufacturing constraints on the diameter and to maximize the generation rate of photon pairs. We end this paper by giving tolerances on the nanofiber diameter in order to give the best experimental approach and give an example of enhancement of the surface nonlinearity using nonlinear layer as a coating on the naked silica nanofiber in order to maximize photon pair generation rate.

\section{Theory and modeling}

\subsection{Architecture of the nanofiber}

Nanofibers are produced by tapering fibers, typically standard telecommunication fibers whose diameter is $125 \mu \mathrm{m}$, to diameters lower than half a micron. They can be obtained via flamebrushing techniques [24,25]. A heater softens a section of this fiber, while its two ends are pulled apart, the resulting object is called a nanofiber. This tapered optical fiber is described in Figure 1. It is a waveguide with circular symmetry made up of a nanofiber connected to two tapers which are necessary to easily and efficiently inject and collect light. Typically, nanofiber diameters are below $1 \mu \mathrm{m}$ over lengths of up to a few centimeters. The small diameter of the tapered sections favors the nonlinear effects, while the non-tapered sections make it possible to connect this tapered fiber with the fibers of the telecommunication networks with very low losses.

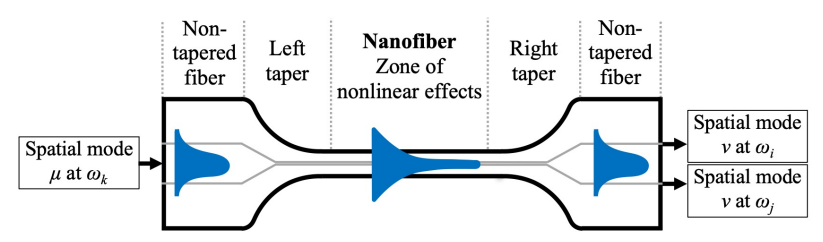

Fig. 1. Principle of SPDC process for photon pair generation by modal phase matching in a tapered silica nanofiber.

For SPDC, pump light is injected as a propagating mode through the left non-tapered fiber (see Fig. 1). Its power is confined inside the fiber core. During its propagation inside the left taper, this mode undergoes a gradually reduced radius and, at the same time, the mode is transformed into a cladding mode. Finally, when it reaches the nanofiber whose diameter is less than one micrometer, light is guided through the "silica-surrounding medium" interface. In this nanofiber section, light is thus sensitive to the surface second-order nonlinearities. We use these nonlinearities to create the correlated photons from the pump beam. These correlated photons propagate as modal fields of the nanofiber, which are gradually transformed by the right taper into core modes of the non-tapered fiber.

Before addressing the modelling of nonlinear propagation inside the nanofiber, we first need a model for the linear propagation. We take into account the fact that the ratio "core diameter over fiber diameter" is kept constant during the pulling of the nanofiber. In the nanofiber section, this original core is thus totally negligible, its diameter being much smaller than the wavelength. Therefore, the nanofiber section is a two-layer medium: a uniform silica rod surrounded by an infinite uniform cladding.

Light propagation in the linear regime in such circular step-index optical fibers has been widely studied in the literature [26]. Ready to use analytic expressions for the light fields and equations for calculating the propagation constants are tabulated in this reference. We rely on 
these exact approaches that take into account the vector nature of the light field. This is mandatory to finely describe light propagation in such small structures whose dimensions are comparable to the optical wavelength.

\subsection{Vectorial mode coupling}

If the linear propagation of light in two-layer nanofibers has been extensively described in the literature, this is not the case of vectorial propagation under second-order nonlinear susceptibility. To develop such a modelling, we decided to rely on an approach previously derived to study vectorial propagation in presence of third-order nonlinearities [27-30]. We use similar notations and formalism to transpose the derivation of these publications to the case of second-order nonlinear susceptibility. Below, we first introduce the notations for the modal fields in the absence of nonlinearity, then, in a second step, we will use these modes as a basis to derive the wave coupling due to the second-order nonlinearity.

\subsubsection{Mode propagation in the linear regime: unperturbed fields}

We first define the unperturbed optical modes as the vectorial modes of the nanofiber without loss and nonlinearity. Silica and cladding are the two isotropic media of this step-index fiber. They are described by refractive index $n$ (which will either be that of silica core, or that of cladding depending on the position). In complex notations, in the Fourier domain and in the absence of sources, they obey the following Maxwell's equations

$$
\left\{\begin{array}{c}
\nabla \wedge \widetilde{\mathbf{E}}_{0}(\mathbf{r}, \omega)=\mathrm{i} \mu_{0} \omega \widetilde{\mathbf{H}}_{0}(\mathbf{r}, \omega), \\
\nabla \wedge \widetilde{\mathbf{H}}_{0}(\mathbf{r}, \omega)=-\mathrm{i} \varepsilon_{0} n^{2}(\mathbf{r}, \omega) \omega \widetilde{\mathbf{E}}_{0}(\mathbf{r}, \omega),
\end{array}\right.
$$

with the Fourier transformation given by

$$
\mathbf{F}(\mathbf{r}, t)=\frac{1}{2 \pi} \int \tilde{\mathbf{F}}(\mathbf{r}, \omega) \mathrm{e}^{-\mathrm{i} \omega t} d \omega
$$

$\mu_{0}$ is the vacuum permeability, $\varepsilon_{0}$ is the dielectric permittivity. $\mathbf{F}$ is either $\mathbf{E}$ the electric field, or $\mathbf{H}$ the magnetic field. Solving Maxwell's equations for the unperturbed fields $\widetilde{\mathbf{E}}_{0}(\mathbf{r}, \omega)$ and $\widetilde{\mathbf{H}}_{0}(\mathbf{r}, \omega)$ results in a complete set of propagating modes. We label these transverse modes by subscript $\eta$. We now identify the wave frequencies by subscript $\omega_{l}$. These modes form an orthonormal basis and can be expressed as follows [26,27]

$$
\begin{gathered}
\widetilde{\mathbf{E}}_{0 \eta}\left(\mathbf{r}, \omega_{l}\right)=\mathrm{e}^{\mathrm{i} \beta_{\eta l z}} \frac{\mathbf{e}_{\eta}\left(\mathbf{r}, \omega_{l}\right)}{\sqrt{N_{\eta l}}}, \\
\widetilde{\mathbf{H}}_{0 \eta}\left(\mathbf{r}, \omega_{l}\right)=\mathrm{e}^{\mathrm{i} \beta_{\eta l z}} \frac{\mathbf{h}_{\eta}\left(\mathbf{r}, \omega_{l}\right)}{\sqrt{N_{\eta l}}},
\end{gathered}
$$

where $\beta_{\eta l}$ is the propagation constant of mode $\eta$ with modal spatial distributions $\mathbf{e}_{\eta}\left(\mathbf{r}, \boldsymbol{\omega}_{l}\right)$ and $\mathbf{h}_{\eta}\left(\mathbf{r}, \omega_{l}\right) . N_{\eta l}$ is the $\eta^{\text {th }}$ guided mode normalization constant at frequency $\omega_{l}$. If subscript $\zeta$ denotes another propagating modal field, $\mathbf{e}_{\zeta}\left(\mathbf{r}, \omega_{l}\right)$ and $\mathbf{h}_{\zeta}\left(\mathbf{r}, \omega_{l}\right)$, then the normalization constants are defined from the orthonormalization relations as [26,27]

$$
\frac{1}{2} \int \mathbf{e}_{\eta}\left(\mathbf{r}, \omega_{l}\right) \wedge \mathbf{h}_{\zeta}^{*}\left(\mathbf{r}, \omega_{l}\right) \cdot \overrightarrow{e_{\mathrm{z}}} d A=\left\{\begin{array}{ccc}
N_{\eta l} & \text { if } & \eta=\zeta \\
0 & \text { if } & \eta \neq \zeta
\end{array},\right.
$$


with * the complex conjugate. Unit vector $\overrightarrow{\mathrm{e}_{\mathrm{z}}}$ is along the nanofiber axis and the integration is conducted over plane $A$ perpendicular to $\overrightarrow{\mathrm{e}_{\mathrm{z}}}$. Expressions for fields $\mathbf{e}_{\eta}$ and $\mathbf{h}_{\eta}$ can be readily found in the literature for the step-index fibers we are studying here (for example in Chapter 12 of [26]).

\subsubsection{Vectorial mode coupling by second-order nonlinearity}

The modes expressed by Eq. (3) form a complete orthonormal basis for any guided optical field propagating in the forward direction. Any electromagnetic fields $\widetilde{\mathbf{E}}\left(\mathbf{r}, \omega_{l}\right)$ and $\widetilde{\mathbf{H}}\left(\mathbf{r}, \omega_{l}\right)$ propagating through the nanofiber at frequency $\omega_{l}$ can thus be written as follows $[26,27]$

$$
\begin{aligned}
& \widetilde{\mathbf{E}}\left(\mathbf{r}, \omega_{l}\right)=\sum_{\eta} a_{\eta}\left(z, \omega_{l}\right) \mathrm{e}^{\mathrm{i} \beta_{\eta l} z} \frac{\mathbf{e}_{\eta}\left(\mathbf{r}, \omega_{l}\right)}{\sqrt{N_{\eta l}}}=\sum_{\eta} a_{\eta}\left(z, \omega_{l}\right) \widetilde{\mathbf{E}}_{0 \eta}\left(\mathbf{r}, \omega_{l}\right)=\sum_{\eta} \widetilde{\mathbf{E}}_{\eta}\left(\mathbf{r}, \omega_{l}\right), \\
& \widetilde{\mathbf{H}}\left(\mathbf{r}, \omega_{l}\right)=\sum_{\eta} a_{\eta}\left(z, \omega_{l}\right) \mathrm{e}^{\mathrm{i} \beta_{\eta l} z} \frac{\mathbf{h}_{\eta}\left(\mathbf{r}, \omega_{l}\right)}{\sqrt{N_{\eta l}}}=\sum_{\eta} a_{\eta}\left(z, \omega_{l}\right) \widetilde{\mathbf{H}}_{0 \eta}\left(\mathbf{r}, \omega_{l}\right)=\sum_{\eta} \widetilde{\mathbf{H}}_{\eta}\left(\mathbf{r}, \omega_{l}\right),
\end{aligned}
$$

coefficients $a_{\eta}$ are such that $\left\|a_{\eta}\right\|$ represents the square root of the $\eta^{\text {th }}$ forward mode power. Maxwell's equations for these perturbed fields $\widetilde{\mathbf{E}}\left(\mathbf{r}, \omega_{l}\right)$ and $\widetilde{\mathbf{H}}\left(\mathbf{r}, \omega_{l}\right)$ are given by

$$
\left\{\begin{array}{c}
\nabla \wedge \tilde{\mathbf{E}}\left(\mathbf{r}, \omega_{l}\right)=\mathrm{i} \mu_{0} \omega_{l} \widetilde{\mathbf{H}}\left(\mathbf{r}, \omega_{l}\right), \\
\nabla \wedge \widetilde{\mathbf{H}}\left(\mathbf{r}, \omega_{l}\right)=-\mathrm{i} \varepsilon_{0} n^{2}\left(\mathbf{r}, \omega_{l}\right) \omega_{l} \widetilde{\mathbf{E}}\left(\mathbf{r}, \omega_{l}\right)-\mathrm{i} \omega_{l} \widetilde{\mathbf{P}}_{N L}\left(\mathbf{r}, \omega_{l}\right),
\end{array}\right.
$$

where $\widetilde{\mathbf{P}}_{N L}$ is the nonlinear electric polarization. We use the formalisms developed in [2730] by constructing function $\mathbf{F}_{\mathrm{c}}$ defined as

$$
\mathbf{F}_{\mathrm{c}}\left(\mathbf{r}, \omega_{l}\right)=\widetilde{\mathbf{E}}_{0 \eta}^{*}\left(\mathbf{r}, \omega_{l}\right) \wedge \widetilde{\mathbf{H}}\left(\mathbf{r}, \omega_{l}\right)+\widetilde{\mathbf{E}}\left(\mathbf{r}, \omega_{l}\right) \wedge \widetilde{\mathbf{H}}_{0 \eta}^{*}\left(\mathbf{r}, \omega_{l}\right) .
$$

Following Turner [27], we relate the unperturbed and perturbed fields using the reciprocal theorem [26]

$$
\frac{\partial}{\partial z} \int \mathbf{F}_{\mathrm{c}}\left(\mathbf{r}, \omega_{l}\right) \cdot \overrightarrow{\mathrm{e}_{\mathrm{z}}} d A=\int \nabla \cdot \mathbf{F}_{\mathrm{c}}\left(\mathbf{r}, \omega_{l}\right) d A
$$

Using decomposition Eq. (5) and modal orthogonality Eq. (4), we immediately identify the left-hand side of the reciprocal theorem

$$
\frac{\partial}{\partial z} \int \mathbf{F}_{\mathrm{c}}\left(\mathbf{r}, \omega_{l}\right) \cdot \overrightarrow{\mathrm{e}_{\mathrm{z}}} d A=4 \frac{\partial a_{\eta}\left(z, \omega_{l}\right)}{\partial z} .
$$

$\nabla . \mathbf{F}_{\mathrm{c}}$, appearing in the second member of Eq. (8) can also be readily calculated using the Maxwell's equations Eq. (1) and Eq. (6) and the formula for the divergence of a cross-product

$$
\nabla . \mathbf{F}_{\mathrm{c}}\left(\mathbf{r}, \omega_{l}\right)=\mathrm{i} \omega_{l} \mathrm{e}^{-\mathrm{i} \beta_{\eta l}} \frac{\mathbf{e}_{\eta \eta}^{*}\left(\mathbf{r}, \omega_{l}\right)}{\sqrt{N_{\eta l}}} \cdot \widetilde{\mathbf{P}}_{N L}\left(\mathbf{r}, \omega_{l}\right),
$$

we replace the two expressions Eq. (9) and Eq. (10) into Eq. (8), and obtain the coupledwave equations

$$
\frac{\partial a_{\eta}\left(z, \omega_{l}\right)}{\partial z}=\frac{\mathrm{i} \omega_{l}}{4} \int \mathrm{e}^{-\mathrm{i} \beta_{\eta !}} \frac{\mathbf{e}_{\eta}^{*}\left(\mathbf{r}, \omega_{l}\right)}{\sqrt{N_{\eta l}}} \cdot \widetilde{\mathbf{P}}_{N L}\left(\mathbf{r}, \omega_{l}\right) d A .
$$

We have as many equations Eq. (11) as frequencies and as transverse modes taken in the field decomposition. Nevertheless, the strength of mode-coupling described by the right-hand 
side of Eq. (11) is significant only for modes that fulfill the phase matching condition. This restricts the number of interacting modes.

Therefore, from now on, we are going to restrict the analysis of one wave at a short wavelength characterized by modal and frequency parameters $(\eta, l)=(\mu, k)$ interacting with two other waves of different but longer wavelengths in the same other transverse mode, thus characterized by parameters $(\eta, l)=(v, i)$ and $(v, j)$.

This situation corresponds to sum-frequency generation SFG, (respectively difference frequency generation, DFG), in which the creation (respectively annihilation) of a photon at frequency $\omega_{k}$ simultaneously occurs with the annihilation (respectively creation) of two photons at frequencies $\omega_{i}$ and $\omega_{j}$. These frequencies are thus related by the energy conservation $\omega_{k}=\omega_{i}+\omega_{j}$. In case no photons are injected in modes $(v, i)$ and $(v, j)$, DFG restricts to the SPDC we are interested in Fig. 1.

SFG, DFG and SPDC are described by a common set of coupled-wave equations. The evolutions of coefficients $a_{\eta}\left(z, \omega_{l}\right)$ are thus expressed by

$$
\begin{aligned}
& \frac{\partial a_{\mu}\left(z, \omega_{k}\right)}{\partial z}=\mathrm{i} \rho^{S F G} a_{v}\left(z, \omega_{i}\right) a_{v}\left(z, \omega_{j}\right) \mathrm{e}^{-\mathrm{i} \Delta \beta z}, \\
& \frac{\partial a_{v}\left(z, \omega_{i}\right)}{\partial z}=\mathrm{i} \rho_{i}^{D F G} a_{\mu}\left(z, \omega_{k}\right) a_{v}^{*}\left(z, \omega_{j}\right) \mathrm{e}^{\mathrm{i} \Delta \beta z}, \\
& \frac{\partial a_{v}\left(z, \omega_{j}\right)}{\partial z}=\mathrm{i} \rho_{j}^{D F G} a_{\mu}\left(z, \omega_{k}\right) a_{v}^{*}\left(z, \omega_{i}\right) \mathrm{e}^{\mathrm{i} \Delta \beta z},
\end{aligned}
$$

where $\Delta \beta=\beta_{\mu k}-\beta_{v i}-\beta_{v j}$ is the phase mismatch, $\rho^{S F G}$ is a normalized overlap integral describing the strength of SFG and $\rho_{i}^{D F G}$ and $\rho_{j}^{D F G}$ integrals for the strengths of DFG. These coefficients can be deduced from Eq. (11). For instance, $\rho^{S F G}$ is expressed by

$$
\rho^{S F G}=\frac{\omega_{k}}{4} \int \frac{\mathbf{e}_{\mu}^{*}\left(\mathbf{r}, \omega_{k}\right)}{\sqrt{N_{\mu k}}} \widetilde{\wp}_{N L}\left(\mathbf{r}, \omega_{k}\right) d A
$$

where

$$
\widetilde{\wp}_{N L}\left(\mathbf{r}, \omega_{k}\right)=\frac{\mathrm{e}^{-\mathrm{i}\left(\beta_{v_{v}}+\beta_{v j}\right) z}}{a_{v}\left(z, \omega_{i}\right) a_{v}\left(z, \omega_{j}\right)} \widetilde{\mathbf{P}}_{N L}\left(\mathbf{r}, \omega_{k}\right),
$$

$\widetilde{\wp}_{N L}$ is the normalized dielectric polarization vector. We can get similar expressions for $\rho_{i}^{D F G}$ and $\rho_{j}^{D F G}$. Nevertheless, it is often more convenient to calculate these two coefficients from $\rho^{S F G}$ from the following Manley-Rowe equations [31]

$$
\left\{\begin{array}{c}
\frac{\rho_{j}^{D F G}}{\rho_{i}^{D F G}}=\frac{\omega_{j}}{\omega_{i}}, \\
\rho^{S F G}=\rho_{i}^{D F G^{*}}+\rho_{j}^{D F G^{*}} .
\end{array}\right.
$$

Therefore, the problem of finding all these three coefficients comes down to finding the only coefficient $\rho^{S F G}$. This is the purpose of the next paragraph.

\subsection{SFG conversion efficiency in silica nanofiber}

To calculate the expression for coefficient $\rho^{S F G}$, we need to precisely detail the waveguide structure. The nanofiber is composed of two layers, silica interior immersed in an infinite isotropic medium which will either be air or a completely inert medium from the point of view of nonlinearity. We therefore completely neglect the nonlinearity of the second medium. We justify this choice because we will only use this second architecture to illustrate how the 
structure can be optimized by the choice of an outside medium with a specific dispersion. We use the axis system depicted in Fig. 2.

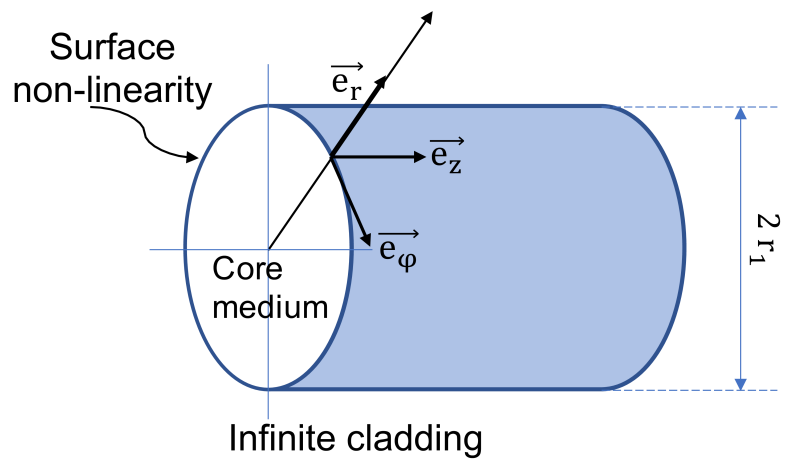

Fig. 2. Axis system in silica nanofiber.

Silica is an isotropic medium generally considered to have $\chi^{(2)}=0$. This affirmation is only valid in an infinite medium under dipolar approximation, two conditions not fulfilled in our geometry. Indeed, optical silica nanofibers present a surface nonlinearity and a bulk secondorder nonlinearity. This bulk nonlinearity originates from the spatial variation of the electric field vector, beyond the dipole approximation, i.e. extended to the quadrupole approximation and more (high order multipoles). This becomes a major mechanism for second-order processes.

The nonlinear electric polarization appearing in expression of $\rho^{S F G}$ in Eq. (13-14) can thus be decomposed into two contributions: the contribution of the bulk and the contribution of second-order nonlinear susceptibility of the surface such as

$$
\widetilde{\mathbf{P}}_{N L}\left(\mathbf{r}, \omega_{k}\right)=\widetilde{\mathbf{P}}^{(2, B u l k)}\left(\mathbf{r}, \omega_{k}\right)+\widetilde{\mathbf{P}}^{(2 s)}\left(\mathbf{r}, \omega_{k}\right) .
$$

\subsubsection{Quadripolar bulk nonlinear polarization}

This polarization originates from the bulk of silica which is a homogeneous and isotropic medium with no free charges. For such a medium, the bulk nonlinear second-order polarization is composed of only two terms as [32,33]

$$
\begin{aligned}
\widetilde{\mathbf{P}}^{(2, B u l k)}\left(\mathbf{r}, \omega_{k}\right)= & \varepsilon_{0} \gamma \nabla\left[\widetilde{\mathbf{E}}_{v}\left(\mathbf{r}, \omega_{i}\right) \widetilde{\mathbf{E}}_{v}\left(\mathbf{r}, \omega_{j}\right)\right] \\
& +\varepsilon_{0} \delta \frac{1}{2}\left(\left[\widetilde{\mathbf{E}}_{v}\left(\mathbf{r}, \omega_{i}\right) . \nabla\right] \widetilde{\mathbf{E}}_{v}\left(\mathbf{r}, \omega_{j}\right)+\left[\widetilde{\mathbf{E}}_{v}\left(\mathbf{r}, \omega_{j}\right) . \nabla\right] \widetilde{\mathbf{E}}_{v}\left(\mathbf{r}, \omega_{i}\right)\right),
\end{aligned}
$$

where parameters $\gamma, \delta$ are the components of the quadrupolar nonlinear susceptibility.

\subsubsection{Surface nonlinear polarization}

The surface nonlinearity arises from a very thin silica layer in the transition region between the silica bulk and the external medium, and, potentially, from external molecules attached to it. This layer is much smaller than the optical wavelength and is thus assimilated to a surface. It is also much smaller than the nanofiber radius. Locally it is thus invariant under a rotation around its normal, that is around $\overrightarrow{\mathrm{e}_{\mathrm{r}}}$. For such a surface, there are only 4 independent non vanishing tensor elements for the second-order susceptibility [34] 


$$
\left\{\begin{array}{c}
\chi_{r r r}^{(2 s)}, \\
\chi_{r z z}^{(2 s)}=\chi_{r \varphi \varphi}^{(2 s)}, \\
\chi_{z r z}^{(2 s)}=\chi_{\varphi r \varphi}^{(2 s)}, \\
\chi_{z z r}^{(2 s)}=\chi_{\varphi \varphi r}^{(2 s)} .
\end{array}\right.
$$

Therefore, the nonlinear second-order surface polarization is also composed of four terms. With the implicit $\left(\mathbf{r}, \omega_{k}\right)$ dependence, we get

where

$$
\tilde{\mathbf{P}}^{(2 s)}=\delta\left(r-r_{1}\right)\left[\tilde{\mathbf{P}}_{1}^{(2 s)}+\tilde{\mathbf{P}}_{2}^{(2 s)}+\tilde{\mathbf{P}}_{3}^{(2 s)}+\tilde{\mathbf{P}}_{4}^{(2 s)}\right]
$$

$$
\left\{\begin{array}{c}
\tilde{\mathbf{P}}_{1}^{(2 s)}\left(\mathbf{r}, \omega_{k}\right)=\varepsilon_{0} \chi_{r r r}^{(2 s)} \overrightarrow{\mathrm{e}_{\mathrm{r}}}\left(\widetilde{\mathbf{E}}_{v}\left(\mathbf{r}, \omega_{i}\right) \cdot \overrightarrow{\mathrm{e}_{\mathrm{r}}}\right)\left(\widetilde{\mathbf{E}}_{v}\left(\mathbf{r}, \omega_{j}\right) \cdot \overrightarrow{\mathrm{e}_{\mathrm{r}}}\right), \\
\tilde{\mathbf{P}}_{2}^{(2 s)}\left(\mathbf{r}, \omega_{k}\right)=\varepsilon_{0} \chi_{r z z}^{(2 s)} \overrightarrow{\mathrm{e}_{\mathrm{r}}}\left(\left(\widetilde{\mathbf{E}}_{v}\left(\mathbf{r}, \omega_{i}\right) \cdot \overrightarrow{\mathrm{e}_{z}}\right)\left(\widetilde{\mathbf{E}}_{v}\left(\mathbf{r}, \omega_{j}\right) \cdot \overrightarrow{\mathrm{e}_{z}}\right)+\left(\widetilde{\mathbf{E}}_{v}\left(\mathbf{r}, \omega_{i}\right) \cdot \overrightarrow{\mathrm{e}_{\varphi}}\right)\left(\widetilde{\mathbf{E}}_{v}\left(\mathbf{r}, \omega_{j}\right) \cdot \overrightarrow{\mathrm{e}_{\varphi}}\right)\right), \\
\tilde{\mathbf{P}}_{3}^{(2 s)}\left(\mathbf{r}, \omega_{k}\right)=\varepsilon_{0} \chi_{z r z}^{(2 s)}\left(\overrightarrow{\mathrm{e}_{z}}\left(\widetilde{\mathbf{E}}_{v}\left(\mathbf{r}, \omega_{i}\right) \cdot \overrightarrow{\mathrm{e}_{\mathrm{r}}}\right)\left(\widetilde{\mathbf{E}}_{v}\left(\mathbf{r}, \omega_{j}\right) \cdot \overrightarrow{\mathrm{e}_{\mathrm{z}}}\right)+\overrightarrow{\mathrm{e}}_{\varphi}\left(\widetilde{\mathbf{E}}_{v}\left(\mathbf{r}, \omega_{i}\right) \cdot \overrightarrow{\mathrm{e}_{\mathrm{r}}}\right)\left(\widetilde{\mathbf{E}}_{v}\left(\mathbf{r}, \omega_{j}\right) \cdot \overrightarrow{\mathrm{e}_{\varphi}}\right)\right), \\
\tilde{\mathbf{P}}_{4}^{(2 s)}\left(\mathbf{r}, \omega_{k}\right)=\varepsilon_{0} \chi_{z z r}^{(2 s)}\left(\overrightarrow{\mathrm{e}_{\mathrm{z}}}\left(\widetilde{\mathbf{E}}_{v}\left(\mathbf{r}, \omega_{i}\right) \cdot \overrightarrow{\mathrm{e}_{\mathrm{z}}}\right)\left(\widetilde{\mathbf{E}}_{v}\left(\mathbf{r}, \omega_{j}\right) \cdot \overrightarrow{\mathrm{e}_{\mathrm{r}}}\right)+\overrightarrow{\mathrm{e}}_{\varphi}\left(\widetilde{\mathbf{E}}_{v}\left(\mathbf{r}, \omega_{i}\right) \cdot \overrightarrow{\mathrm{e}_{\varphi}}\right)\left(\widetilde{\mathbf{E}}_{v}\left(\mathbf{r}, \omega_{j}\right) \overrightarrow{\mathrm{e}_{\mathrm{r}}}\right)\right),
\end{array}\right.
$$

and with $\delta\left(r-r_{1}\right)$ is the Dirac distribution centered on the nonlinear surface $r_{1}$ (Fig. 2).

In Eq. (20), we have omitted the implicit dependence of the susceptibilities with frequencies $\left(\omega_{i}, \omega_{j}\right)$, however this dependence makes $\chi_{z r z}^{(2 s)}$ different from $\chi_{z z r}^{(2 s)}$ away from degeneracy. At degeneracy, $\omega_{i}=\omega_{j}$, these two coefficients are equal, and these equations reduce to the previously studied SHG case [34,35].

We have now all the equations needed to predict the evolutions of the optical fields for SFG or DFG, by replacing the expressions for the polarization, Eq. (17-20) in the expressions for the efficiencies, Eq. (13-15), and solving the set of coupled-wave equations, Eq. (12); the analytical expressions for the optical fields being tabulated from the literature (Chapter 12, table 12.3 in [26] for instance). We can thus solve these equations with the desired boundary conditions, i.e. the field values at the nanofiber input.

A semi classical approach cannot describe the full quantum properties of SPDC but can perfectly be used to calculate the number of generated pairs we use to evaluate the performance of the proposed source.

\subsection{Generation rate of photon pairs}

Referring to the notation used to write Eq. (12), we now consider that a pump beam at frequency $\omega_{k}$ is injected at the nanofiber input, while the photon pair is collected at frequencies $\omega_{i}$ and $\omega_{j}$ (Fig. 1). As a matter of principle, pairs of photons must be created one by one, in the parametric fluorescence regime with no injected light at frequencies $\omega_{i}$ and $\omega_{j}$. Therefore, the conversion efficiency is low, and the pump is considered to be undepleted. The system of Eq. (12) resolves to the only Eq. (12b) and (12c). Quantum models of the photon pair generation in SPDC crystals have been developed [36-38] to describe the full quantum properties of the generated photons. As we are here interested in the number of generated photons, we prefer the semi-classical derivation of quantum generation of photon pairs [39-41], that presents a closer connection to the nonlinear wave mixing equations we used until now. Number $N_{p h}$ of photon pairs emitted per unit of time at the output of a nanofiber of length $L$ in the two $v^{\text {th }}$ spatial modes is

$$
N_{p h}=\int G_{v}(L, \omega) d \omega,
$$


Where $G_{v}(L, \omega)$ is the spectral density of photons generated in either of the two $v^{\text {th }}$ spatial modes at frequency $\omega$. It is given by

$$
G_{v}(L, \omega)=g^{2} L^{2} \operatorname{sinc}\left(\sqrt{\left(\frac{\Delta \beta}{2}\right)^{2}-g^{2} L}\right)^{2},
$$

with parameter $g$ defined as a function of the pump power $P_{\text {pump }}$ as

$$
g^{2}=\rho_{i}^{D F G} \rho_{j}^{D F G^{*}} P_{p u m p} \quad \text { with } \quad P_{\text {pump }}=\left\|a_{\mu}\left(z, \omega_{k}\right)\right\|^{2} .
$$

Eq. (22) takes into account the fact that the argument of the unnormalized sinc function may become purely imaginary in which case the sinc is to be changed into an hyperbolic sinc function.

Using the Manley-Rowe equations (Eq. 15), parameter $g$ can be expressed as

$$
g^{2}=\frac{\lambda_{i} \lambda_{j}}{\left(\lambda_{i}+\lambda_{j}\right)^{2}}\left|\rho^{S F G}\right|^{2} P_{\text {pump }}
$$

Eq. (24) suggests two approaches to compute the generation rate $N_{p h}$, Eq. (21):

- One can perform a second harmonic generation experiment to directly measure $\rho^{S F G}$, deduce $\rho_{i}^{D F G}$ and $\rho_{j}^{D F G}$ from the Manley-Rowe equations, Eq. (15), and then report their values into Eq. (21). This SHG experiment shall be conducted with exactly the same modes as those intended to be used for the SPDC generation of photon pairs.

- Alternatively, one can compute the value for $\rho^{S F G}$ starting from Eq. (13) in which we inject the expressions for the nonlinear polarizations, Eq. (17) and $(19,20)$.

In the following examples, we follow this second approach that requires the knowledge of the second-order susceptibilities.

\section{Spectral density of photon pair generation for various architectures}

\subsection{General parameters}

\subsubsection{Interacting modes}

For each of the following examples, we consider the case of a pump beam at wavelength $\lambda_{k}=$ $775 \mathrm{~nm}$ so that, in the vicinity of degeneracy, the pairs of photons are created around $\lambda_{i, j} \simeq$ $1550 \mathrm{~nm}$. These wavelengths are compatible with standard optical communication networks. For the output spatial modes $v$ we select the fundamental $H E_{11}$ nanofiber mode because this is the one that will most easily couple into the $H E_{11}$ mode of standard telecom fibers.

To maximize the emission rate $N_{p h}$ (Eq. 21), the phase mismatch $\Delta \beta$ should be minimized. We relate this phase mismatch $\Delta \beta$ to the effective mode index. The effective mode index and the propagation constants in a step-index two-layered circular waveguide are easily computed from expressions given in [26] (table 12.4 chapter 12). Typically, one should keep $|\Delta \beta L| \leq \pi$. Due to effective index dispersion, perfect phase matching, $\Delta \beta L=0$, can only be obtained in nanofibers by modal phase matching, that is by using an input pump mode, mode $\mu$, that differs from the output modes $H E_{11}$, modes $v$.

To set this idea on a simple example, we have just to remind that perfect phase matching is simply equivalent to assure $n_{e f f}\left(\lambda_{k}\right)=n_{e f f}\left(\lambda_{i, j}\right)$, that is why we plotted, in the following Fig. 3, the refractive indices at $\lambda_{k}=775 \mathrm{~nm}$ (full lines) and at $\lambda_{i, j} \simeq 1550 \mathrm{~nm}$ (dashed lines) for the five lowest order modes. 


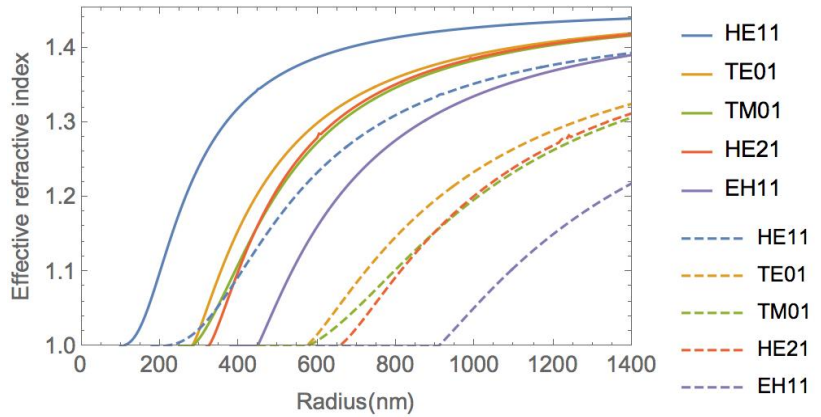

Fig. 3. Refractive indices of the five lowest order modes at $1550 \mathrm{~nm}$ (in dashed lines) and $775 \mathrm{~nm}$ (in full lines) versus the radius of a naked nanofiber. Phase matching in this radius range only exists between $H E_{11}$ at $1550 \mathrm{~nm}$ and $T E_{01}$ or $T M_{01}$ or $H E_{21}$ at $775 \mathrm{~nm}$.

One sees that for the radius range of this figure, $H E_{11}$ at $\lambda_{i, j} \simeq 1550 \mathrm{~nm}$ can only be phase matched to the pump at $\lambda_{k}=775 \mathrm{~nm}$ in one of the three lowest orders $T E_{01}, T M_{01}$ and $H E_{21}$. For the range considered, there are no other phase matchings. We are not interested in phase matching for higher radii as the efficiencies will be lower due to a lower mode confinement. Conversely, any of these pump modes is only phase matched with $H E_{11}$ and not to any higher order modes: the output photons will thus be created in these $H E_{11}$ modes only.

These are thus the only three possible pump modes we are going to consider in the following.

Contrary to modes $T E_{01}$ and $T M_{01}$, modes $H E_{11}$ and $H E_{21}$ are polarization degenerated. We denote this degeneracy by superscripts $e^{e v e n}$ and ${ }^{o d d} . H E_{11}^{o d d}$ is obtained from $H E_{11}^{\text {even }}$ by a rotation of $\pi / 2$ around the nanofiber axis (Fig. 4). They share the same effective refractive index for a perfectly circular nanofiber (the reason for which we did not take into account the mode polarization when plotting Fig. 3). Similarly, $H E_{21}^{\text {odd }}$ is obtained from $H E_{21}^{\text {even }}$ by a rotation of $\pi / 4$ around the nanofiber axis and both these modes have the same effective refractive index (Fig. 4). 

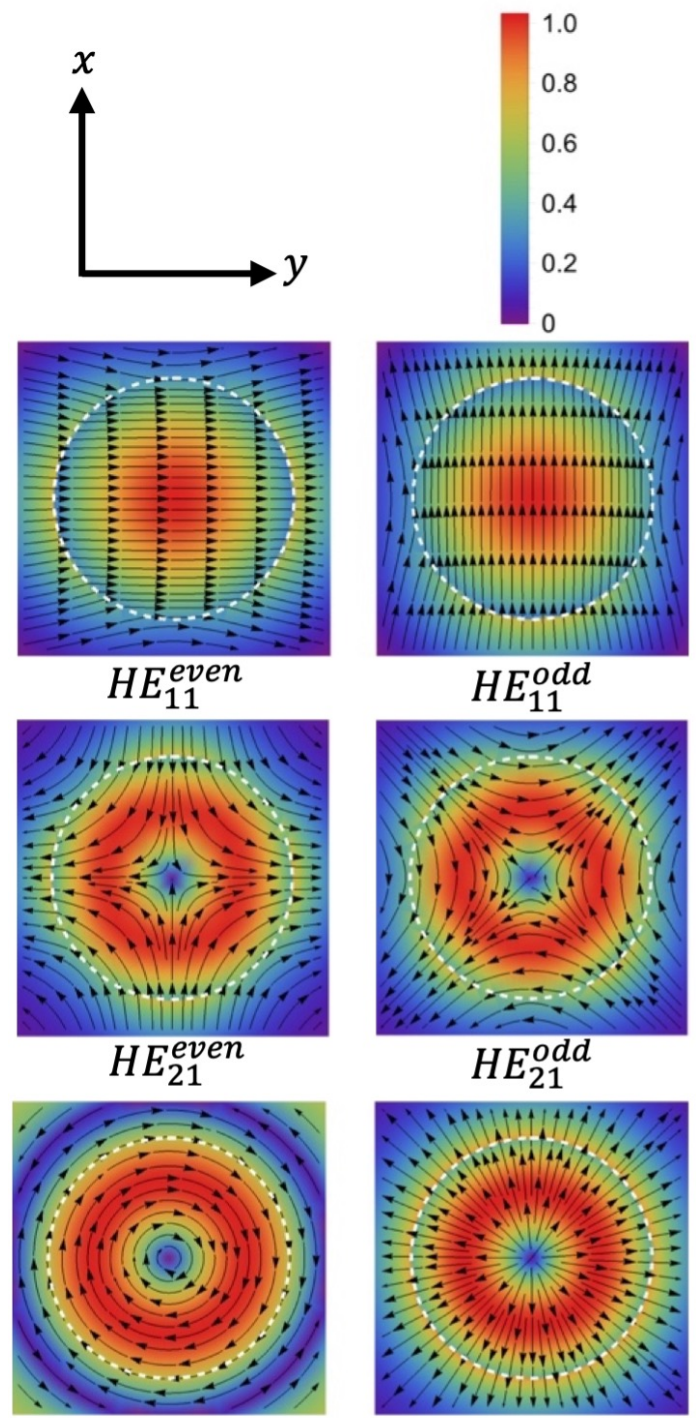

$T E_{01}$

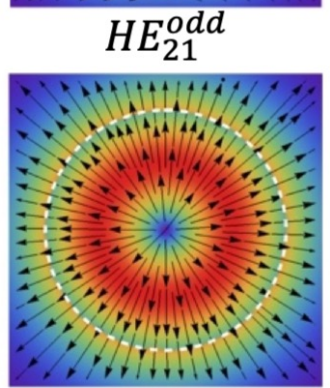

$T M_{01}$

Fig. 4. Structures of the in-plane components of the electric fields for the different modes. The axes $(\mathbf{x}, \mathbf{y})$ are purely arbitrary in a perfect circular fiber. The dashed white circles represent the surface of the naked silica nanofiber. The background color indicates the strength of the in-plane electric field component. For this example, the nanofiber radius is $0.5 \mu \mathrm{m}, H E_{11}$ wavelength is set at $1550 \mathrm{~nm}$, and the pump modes are at $775 \mathrm{~nm}$.

The phase matching conditions, shown by the intersects of the effective refractive index curves in Fig. 3, do not say anything about the strength of the overlap integral $\rho^{S F G}$ and thus about the fluorescence efficiencies and polarization states. It just indicates that, potentially, for any of these pump modes, the output photons, if they exist, can only be in the $H E_{11}$ modes.

In the undepleted pump analysis we conduct here, the amplitudes of the output fields are governed by Eq. (12b) and (12c). However, we do have as many such equations as there are fluorescence processes. The overlap integrals $\rho_{j}^{D F G}$ now depend on the considered set of polarizations. For sake of clarity we omit to indicate the dependence in $z$, and with straightforward notations we easily generalize Eq. (12b) and (12c) into 


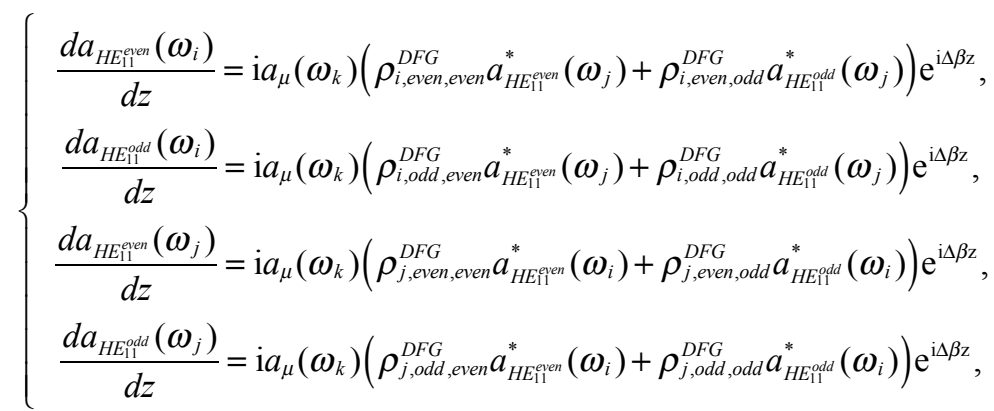

The overlap integrals are proportional to the Sum Frequency Generation overlap integrals, and thus proportional to the nonlinear polarization terms. They are themselves proportional to the product of the two electric components or to their derivatives. The parity dependence of these fields is fully separable from the radial dependence and is wavelength independent. Therefore, the polarizations do not depend on the order of appearance of these parity dependences in the equations. We thus state that

$$
\rho_{i \text { or } j, \text { odd }, \text { even }}^{D F G}=\rho_{i \text { or } j, \text { even, odd }}^{D F} .
$$

In Eq. (25), mode parameter $\mu$ represents any of the possible input modes $T E_{01}, T M_{01}$ or $H E_{21}$. In the following we analyze the values taken by the output integrals for each of these possible input modes.

\subsubsection{Configuration with the $T E_{01}$ pump beam}

Taking into account the rotational symmetry of mode $T E_{01}$, and then reporting the expressions, given in the literature [26], for the electric fields in the overlap integrals we obtain

$$
\rho_{i \text { or } j, \text { even,even }}^{D F G}=\rho_{i \text { or } j, \text { odd }, \text { odd }}^{D F G}=0 .
$$

It is also possible to show that at degeneracy, i.e. when $\omega_{i}=\omega_{j}$, the overlap integrals $\rho_{i \text { or } j, \text { even }, o d d}^{D F G}=\rho_{i \text { or } j, \text { odd,even }}^{D F G}$ also vanish. Outside degeneracy, only the bulk nonlinear polarization contributes to these overlap integrals.

We conclude that at degeneracy no photons are created. Outside degeneracy, the two photons are orthogonally polarized. Indeed, if a photon is detected at frequency $\omega_{i}$ in the $H E_{11}^{\text {even }}$ mode, that means that the coupling is described by Eq. (25a) and (25d). Therefore, the other at frequency $\omega_{j}$ is in the $H E_{11}^{\text {odd }}$ mode. Similarly, if a photon is detected at frequency $\omega_{i}$ in the $H E_{11}^{\text {odd }}$ mode, then Eq. (25b) and (25c) are at the origin of the creation of the photon pairs and the second photon, at frequency $\omega_{j}$, is in the $H E_{11}^{\text {even }}$ mode.

Detecting the photons in these modes is quite easy: a beam splitter polarizing cube aligned along the $\mathbf{y}$ - and $\mathbf{x}$-axes nearly perfectly splits the content of the $H E_{11}^{\text {even }}$ and $H E_{11}^{\text {odd }}$ modes, see Fig. 4. A rotation of this cube around the $\mathbf{z}$-axis does not change the orthogonality of the polarization of the two photons due to the rotational symmetry of $T E_{01}$ around this axis.

For silica, as will be shown in paragraph 3.2, the bulk contribution is lower than the surface contribution. This configuration thus leads to efficiencies lower than with the other pump modes that also involve the contribution of surface. We thus do not analyze in more detail hereafter this configuration involving the $T E_{01}$ pump mode.

\subsubsection{Configuration with the $T M_{01}$ pump beam}

Due to symmetry considerations, and from the analysis of the overlap integrals we can show that

$$
\begin{aligned}
& \rho_{i \text { or } j, \text { even, odd }}^{D F G}=\rho_{i \text { or } j \text {,odd }, \text { even }}^{D F G}=0, \\
& \rho_{i \text { or } j, \text { even, }, \text { ven }}^{D F G}=\rho_{i \text { or } j, \text { odd }, \text { odd }}^{D F G} \neq 0 .
\end{aligned}
$$


One thus concludes that if a $T M_{01}$ pump beam is injected in the nanofiber, then the two photons are produced in the same mode, either $H E_{11}^{\text {even }}$ or $H E_{11}^{\text {odd }}$. As for the previous configuration, rotating the analyzing polarizing cube around the $\mathbf{z}$-axis does not change this fact.

We will compute values for the overlap integrals in section 3.2 for this configuration.

\subsubsection{Configurations with the $H E_{21}$ pump beam}

Whatever the selected input mode $H E_{21}^{\text {odd }}$ or $H E_{21}^{\text {even }}$, we get with the expressions for the electric fields given in the literature [26]

$$
\begin{aligned}
& \rho_{i \text { or } j \text {,even,odd }}^{D F G}=\rho_{i \text { or } j, \text { odd }, \text { even }}^{D F G}=0, \\
& \rho_{i \text { or } j, \text { even, }, \text { even }}^{D F G}=-\rho_{i \text { or } j \text { odd }, \text { odd }}^{D F G} \neq 0 .
\end{aligned}
$$

Therefore, one concludes that if a photon is detected at frequency $\omega_{i}$ in the $H E_{11}^{e v e n}$ mode (respectively $H E_{11}^{\text {odd }}$ mode) then the other photon at frequency $\omega_{j}$ is in the $H E_{11}^{\text {even }}$ mode (respectively $H E_{11}^{\text {odd }}$ ) as reflected by the following set of equations, deduced from Eq. (25)

$$
\begin{aligned}
& \int \frac{d a_{H E_{11}^{\text {oev }}}\left(\omega_{i}\right)}{d z}=\mathrm{i} a_{\mu}\left(\omega_{k}\right) \rho_{i, \text { even, even }}^{\text {DFG }} a_{H E_{11}^{\text {oeen }}}^{*}\left(\omega_{j}\right) \mathrm{e}^{\mathrm{i} \Delta \beta z}, \\
& \frac{d a_{H E_{1}^{\text {odd }}}\left(\omega_{i}\right)}{d z}=-\mathrm{i} a_{\mu}\left(\omega_{k}\right) \rho_{i, \text { even,even }}^{D F G} a_{H E_{1}^{\text {odd }}}^{*}\left(\omega_{j}\right) \mathrm{e}^{\mathrm{i} \Delta \beta z}, \\
& \frac{d a_{H E_{1 !}^{\text {eqen }}}\left(\omega_{j}\right)}{d z}=\mathrm{i} a_{\mu}\left(\omega_{k}\right) \rho_{j, \text { even, }, \text { ven }}^{D F G} a_{H E_{11}^{\text {even }}}^{*}\left(\omega_{i}\right) \mathrm{e}^{\mathrm{i} \Delta \beta z}, \\
& \frac{d a_{H E_{11}^{\text {odd }}}\left(\omega_{j}\right)}{d z}=-\mathrm{i} a_{\mu}\left(\omega_{k}\right) \rho_{j, e v e n, \text { vern }}^{D F G} a_{H E_{11}^{\text {odd }}}^{*}\left(\omega_{i}\right) \mathrm{e}^{\mathrm{i} \Delta \beta z} .
\end{aligned}
$$

The fact that the overlap integrals, Eq. (29b) are opposite in sign highlights the importance of the choice of the polarization basis. As explained above, the polarization basis corresponds to the orientation of the polarizers used for analyzing the photon polarizations. Using a beam splitter polarizing cube aligned along the $\mathbf{y}$-and $\mathbf{x}$-axes nearly perfectly splits the content of the $H E_{11}^{\text {even }}$ and $H E_{11}^{\text {odd }}$ modes. We could for instance rotate the cube by $45^{\circ}$ around the $\mathbf{z}$-axis and thus detect the output in the $\left(H E_{11}^{\text {even }}+H E_{11}^{\text {odd }}, H E_{11}^{\text {even }}-H E_{11}^{\text {odd }}\right)$ analysis basis. The analysis should thus be done in this new basis. From Eq. (30), we immediately get

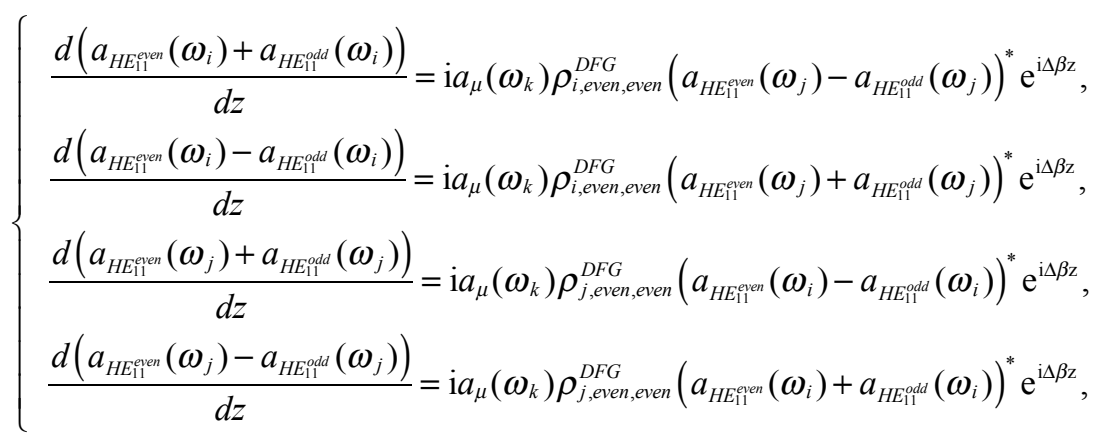

We thus conclude, that if we analyze the polarization of the output photons in this new basis, the two output photons are orthogonally polarized (that is they are either coupled by Eq. (31a) and (31d), or by Eq. (31b) and (31c).

We present some numerical examples in next section in the $\left(H E_{11}^{\text {even }}, H E_{11}^{\text {odd }}\right)$ basis. The values given in next section correspond to a single fluorescence process among the two possible processes: for instance, to the coupled-wave equations Eq. (30a) and (30c), and we do not consider those created by Eq. (30b) and (30d). Considering both processes would simply double 
the generation rate. These values are computed for an input pump power of $1 \mathrm{~W}$. However, because parameter $g L \ll 1$, spectral density $G_{v}(L, \omega)$ is proportional to this pump power with a very good approximation so that extrapolation to another pump power is straightforward.

\subsection{Silica-air architecture}

\subsubsection{Spectral density}

As a first example we consider a silica nanofiber suspended in air. The nonlinear surface and bulk susceptibilities are reported in the literature from a SHG experiment from a wavelength of $1064 \mathrm{~nm}$, see Table $1[34,35]$. Because the wavelengths we consider here are far from resonances, and according to Miller's rule [33], these susceptibilities vary by less than $4 \%$ over the wavelength domain considered here. Thus, hereafter, we consider that these second-order susceptibilities are constant and equal to their values at degeneracy.

Table 1. Surface and quadripolar second-order susceptibility components $\left(\mathrm{pm}^{2} / \mathrm{V}\right) \cdot[34,35]$

\begin{tabular}{ccccc}
\hline$\chi_{r r r}^{(2 s)}$ & $\chi_{r z z}^{(2 s)}=\chi_{r \varphi \varphi}^{(2 s)}$ & $\chi_{z r z}^{(2 s)}=\chi_{z z r}^{(2 s)}=\chi_{\varphi r \varphi}^{(2 s)}=\chi_{\varphi \varphi r}^{(2 s)}$ & $\gamma$ & $\delta$ \\
\hline $6.310^{3}$ & $7.710^{2}$ & $7.910^{2}$ & $-3.910^{2}$ & $7.810^{2}$ \\
\hline
\end{tabular}

In Fig. 5, we plotted the spectral density $G_{v}(L, \omega)$ for, a nanofiber length $L=100 \mu \mathrm{m}$. The input modes are either $T M_{01}$ or $H E_{21}^{\text {even }}$.

The dashed red curve follows the locations of the phase matched wavelengths: for a given radius, the two ordinates $\lambda_{i, j}$ correspond to the two output wavelengths of modes $v$ that are phase matched with the input pump mode at $775 \mathrm{~nm}$, that is for which $\Delta \beta=0$. 

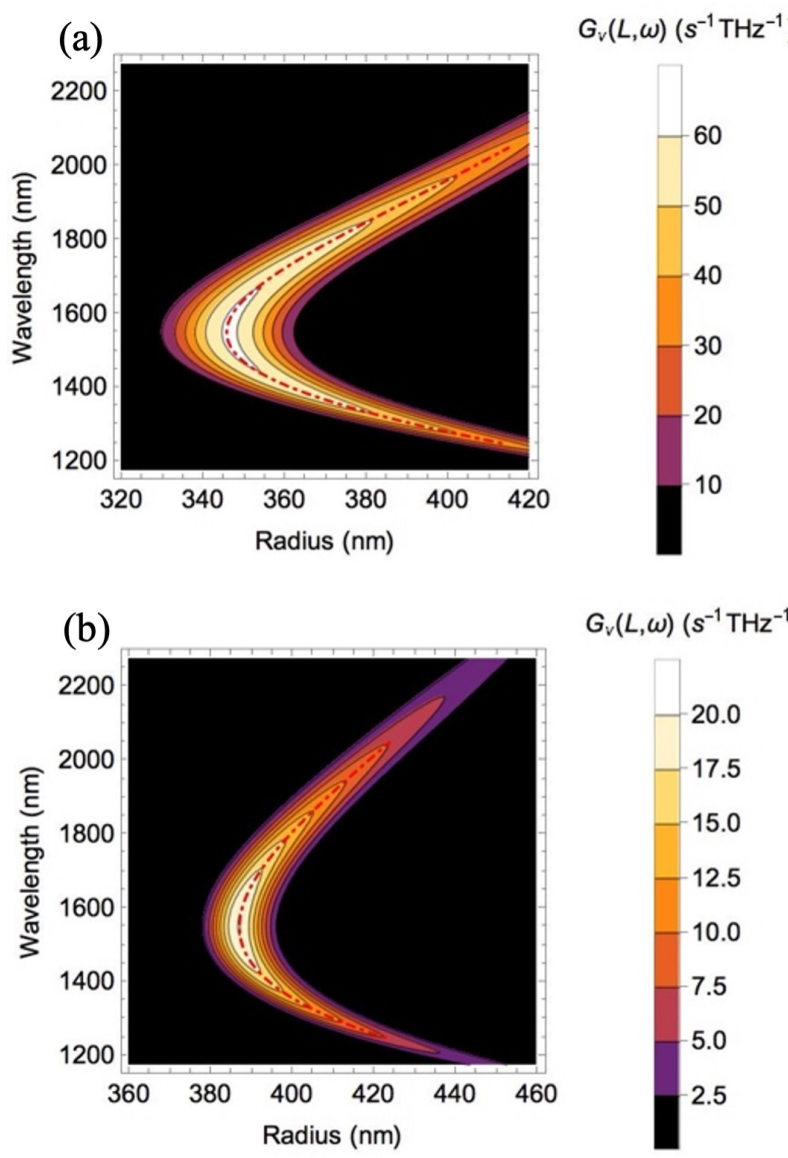

Fig. 5. Spectral density for $1 \mathrm{~W}$ pump power generated in the fundamental mode $H E_{11}$ for a $100 \mu \mathrm{m}$ long silica nanofiber in air. (a) for a $T M_{01}$ input mode. (b) for a $H E_{21}$ input mode. The dashed red curves represent the location of the phase matched wavelengths (see text).

As anticipated, for each wavelength, the maximum of the spectral density is approximately located on this phase matching position. This comes from the dependence of $G_{v}(L, \omega)$ that decreases quickly as soon as $\Delta \beta$ departs from zero because of the sinc function. Nevertheless, a closer look at these curves would reveal that the maximum of the spectral density is slightly shifted from these curves, either in the direction of the low fiber radii, or large fiber radii, depending on the configuration. The reason is that $\Delta \beta$ does not appear in the expression for parameter $g$. Thus, the maximum of $g$ can be set anywhere in the "wavelength-radius" plane. This dependence of parameter $g$ with wavelength and radius is also responsible for the decay of the maximum of the spectral density when one moves away from the wavelength of $1550 \mathrm{~nm}$. As shown by Eq. (22), this maximum, approximatively located along the red curve, $\Delta \beta=0$, scales quadratically versus the nanofiber length $L$. Increasing this length to increase the pair rate is thus tempting, however we will see in next section that the nanofiber radius uniformity somehow limits this possible increase.

Spectral density is higher for the $T M_{01}$ pump than for the $H E_{21}$ pump. This density also decays more slowly in this configuration when we move away from the location of the phasematched wavelengths (dashed red curves in Fig. (5)). This makes this configuration more attractive. This decay versus the fiber radius comes from the variation of $\Delta \beta L$ : to keep a 
decrease bellow $50 \%$ we should typically have $|\Delta \beta L|<2.8$, corresponding to $\operatorname{sinc}(0.5 \Delta \beta L)^{2}=0.5$ see Eq. (22).

\subsubsection{Photon pair rate}

Eq. (21) represents number $N_{p h}$ of photon pairs emitted per unit of time in each one of the output modes (corresponding to the two branches of the spectral density plotted in Fig. 5). Equivalently, $N_{p h}$ represents the photon pairs emitted per unit of time if we limit the integration over any one of these two branches in order to avoid counting the two photons of the same pair. Hereafter, for the purpose of the example, we suppose that no spectral filter is present and thus we conduct the integration over the full range, $0.5 \omega_{\mu}$ to infinity, that is we compute the total number of emitted pairs. In Fig. 6, we plotted this total number of pairs generated per second for a pump power of $1 \mathrm{~W}$ in nanofibers of different lengths for the two configurations. These figures confirm that the configuration with a $T M_{01}$ pump mode is very interesting as it provides a photon pair generation rate three times larger than for the other configuration.
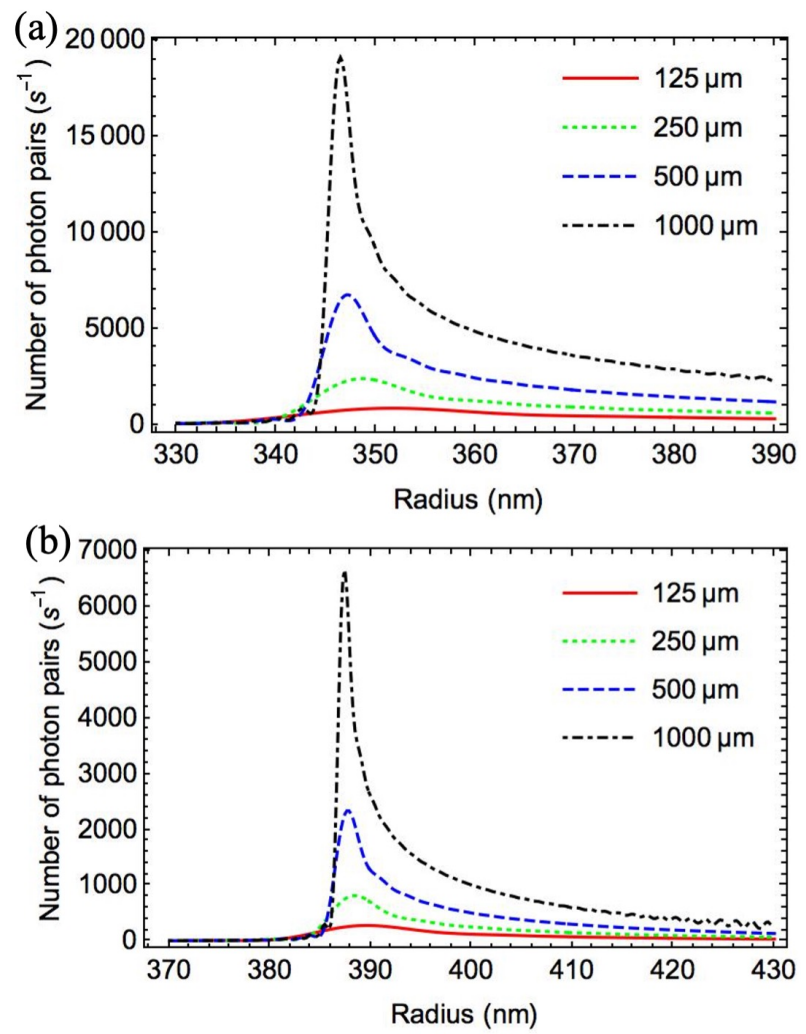

Fig. 6. Number of photon pairs generated per second in a silica nanofiber suspended in air for different lengths and for a $1 \mathrm{~W}$ input power: (a) $T M_{01}$ input mode; (b) $H E_{21}$ input mode.

One notices that these generation rates are all the more peaked that the nanofiber is long. For instance, for a $T M_{01}$ input mode and for a $1000 \mu \mathrm{m}$ long nanofiber, the peak width is about $3 \mathrm{~nm}$ in radius. Setting the operating point of the photon pair source around the top of these curves, that is around degeneracy, imposes severe constraints on the nanofiber fabrication process. Diameter measurements made on silica nanofibers have already demonstrated the high reproducibility of their waist and very good diameter uniformity over large lengths [42-46]. For instance, monitoring the diameter of $300 \mathrm{~mm}$ long nanofibers proved to produce nanofibers with a manufactured diameter varying by less than $5 \mathrm{~nm}$ from the targeted diameter [42]. Would the diameter not exactly be the targeted diameter, one also would have the possibility to slightly 
tune the pump wavelength to shift the operating point on top of the peak of Fig. 6. Typically, if one changes the radius by $5 \mathrm{~nm}$, the pump wavelength for degenerate phase matching is shifted from $775 \mathrm{~nm}$ to $786 \mathrm{~nm}$. The problem is not crucial away from degeneracy.

\subsubsection{Limitations due to the fabrication process}

Photon pair sources manufactured with much longer nanofibers, a few centimeters or a few tens of centimeters, are also feasible. However, the prediction of the photon pair rates is more complex for these longer fibers. Indeed, with longer fibers, the peaks of the generation rates versus the nanofiber radius become very narrow, below $1 \mathrm{~nm}$. This comes from the width of the spectral density versus the radius that decreases proportionally to the inverse of fiber length $L$ as explained in the previous section. Because a nanofiber is never perfect, radius fluctuations along the nanofiber axis induce significant fluctuations in $\Delta \beta$. Typically, derivation of Eq. (22) fails if the nanofiber length $L$ becomes larger than a maximum length $L_{\max }$ defined as [47]

$$
L_{\max } \simeq \frac{2.8}{\frac{\partial \Delta \beta}{\partial r} \delta r_{\max }},
$$

with $\delta r_{\max }$ the maximum deviation of the radius from the radius at phase matching.

The derivative $\partial \Delta \beta / \partial r$ can be computed from the propagation constants that are given in the literature [26]. We obtain

- $T M_{01}$ input, $\partial \Delta \beta / \partial r=2.610^{12} \mathrm{~m}^{-2}$,

- $H E_{21}$ input, $\partial \Delta \beta / \partial r=5.110^{12} \mathrm{~m}^{-2}$.

From the literature, we found that $\delta r_{\max }=1 \mathrm{~nm}$ is realistic. Therefore, for our two configurations and a $1 \mathrm{~W}$ input power, we obtained

- $T M_{01}$ input, $L_{\max } \simeq 1.1 \mathrm{~mm}$ so that $N_{p h} \approx 22000$ pairs $/ \mathrm{s}$,

- $H E_{21}$ input, $L_{\max } \simeq 0.55 \mathrm{~mm}$ so that $N_{p h} \approx 2700$ pairs $/ \mathrm{s}$.

Thus, working with $1 \mathrm{~mm}$ long nanofibers to produce photon pair rates of more than 10000 pairs/s with a $1 \mathrm{~W}$ input power seems to be within reach of today's manufacturing processes.

Working with longer nanofibers is of course possible and could be desirable. Radius fluctuations along the nanofiber then enlarges and flattens the spectral density curves, Fig. 5, and so for the photon rate curves, Fig. 6. These fluctuations thus reduce the photon rates. A prediction of these photon rates can still be made, by replacing the sinc function in Eq. (22) by an integral equation of $\Delta \beta$ along the nanofiber length as reported in reference [47]. This calculation is nevertheless only possible if one knows the radius fluctuation of the nanofiber.

\subsection{Phase matching optimization: example of silica-PTFE architecture}

As just explained, the fluctuation of the fiber radius along its length severely limits the spectral density of the pair rate. A known way to circumvent the influence of the fluctuations of fabrication parameters of waveguides is known as noncritical phase matching [48]. The idea is to find a structure that minimizes parameter $\partial \Delta \beta / \partial r$.

We propose to minimize this parameter by a deposit of an embedding medium onto the silica nanofiber. This medium extends from $r_{1}$ to infinity (Fig. 2). For the purpose of the present demonstration, we assume that this medium is without loss, and that its surface second-order nonlinearity is negligible compared to the ones of the silica, and that it does not affect the silica nonlinear coefficient. Its only influence is on the mode shapes and propagation constants through its refractive index. To take a realistic dispersion for the refractive index, we take the one of a PTFE polymer: Teflon ${ }^{\circledR A F} 1300$ [49].

In Fig. 7, we have plotted the spectral density curves of photon pair generation in a silicaPTFE architecture for the two possible input modes $T M_{01}$ and $H E_{21}$. To allow a direct 
comparison with the previously discussed case of silica in air, Fig. 5, the pump power is set to $1 \mathrm{~W}$ and the length is $L=100 \mu \mathrm{m}$.
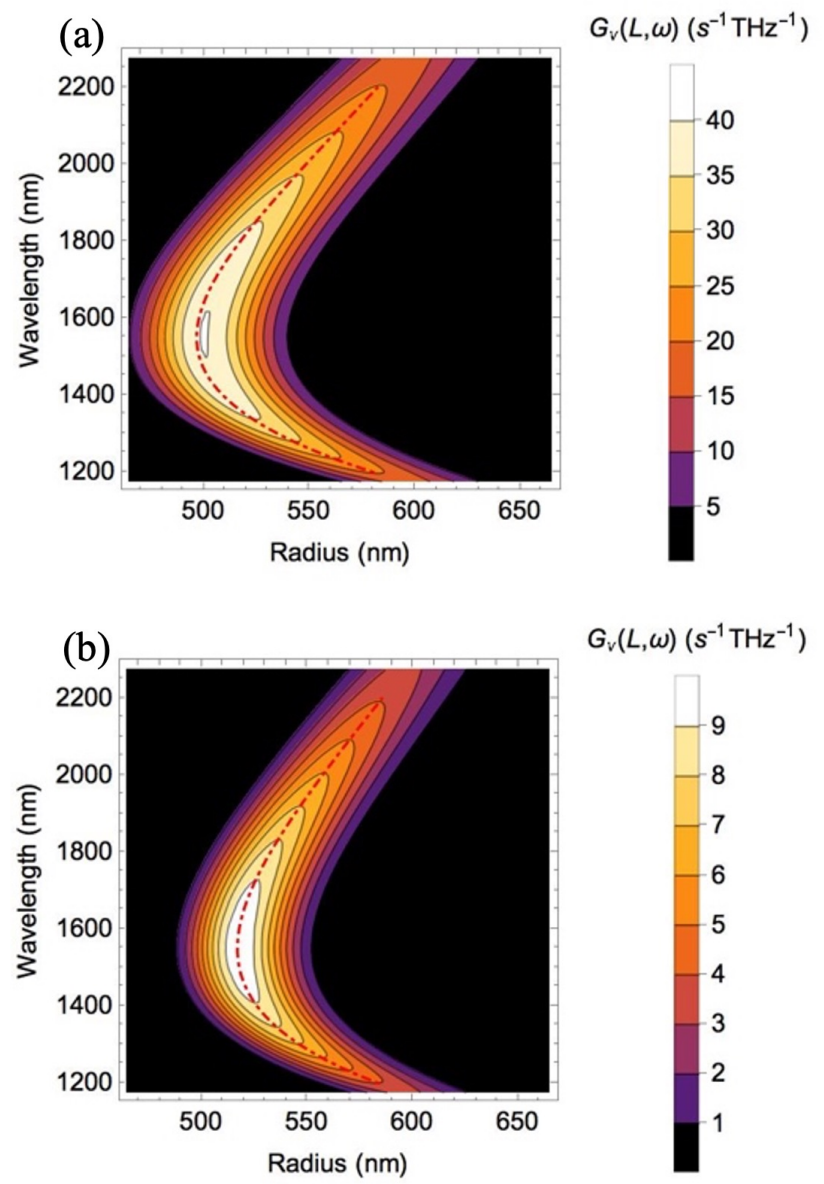

Fig. 7. Spectral densities for a $1 \mathrm{~W}$ pump power generated in the fundamental mode $H E_{11}$ of a $100 \mu \mathrm{m}$ long silica nanofiber embedded in PTFE. (a) $T M_{01}$ input mode. (b) $H E_{21}$ input mode. The dashed red curves represent the location of the phase matched wavelengths (see text).

Compared to Fig. 5, the radius at degeneracy is increased, as a consequence of the less stringent confinement due to the surrounding medium. As a result, one could expect a strong decrease of the spectral density, which is not the case. The nonlinear polarization and pump field decrease as a result of this deconfinement, which indeed decreases the integrant appearing in the expression for $\rho^{S F G}$, Eq. (13). However, for surface nonlinearity the integration is conducted along the nanofiber circumference, that is, proportional to the nanofiber radius. The decrease of the strength of the field is thus compensated by this integration over a larger circumference.

As desired, the consequence of embedding the nanofibers is an enlargement of the spectral density curves that can be quantized by computing parameter $\partial \Delta \beta / \partial r$. We computed

- $T M_{01}$ input, $\partial \Delta \beta / \partial r=1.210^{12} \mathrm{~m}^{-2}$,

- $H E_{21}$ input, $\partial \Delta \beta / \partial r=1.410^{12} \mathrm{~m}^{-2}$.

Once again, for $\delta r_{\max }=1 \mathrm{~nm}$ we obtained

- $T M_{01}$ input, $L_{\max } \simeq 2.4 \mathrm{~mm}$ so that $N_{p h} \approx 78000 \mathrm{pairs} / \mathrm{s}$,

- $H E_{21}$ input, $L_{\max } \simeq 2.0 \mathrm{~mm}$ so that $N_{p h} \approx 15000$ pairs/s. 
For sake of comparison with the structure of silica nanofiber in air, see Fig. 6, we plotted the evolution of the photon rate versus the nanofiber radius in Fig. 8 for these optimized structures.
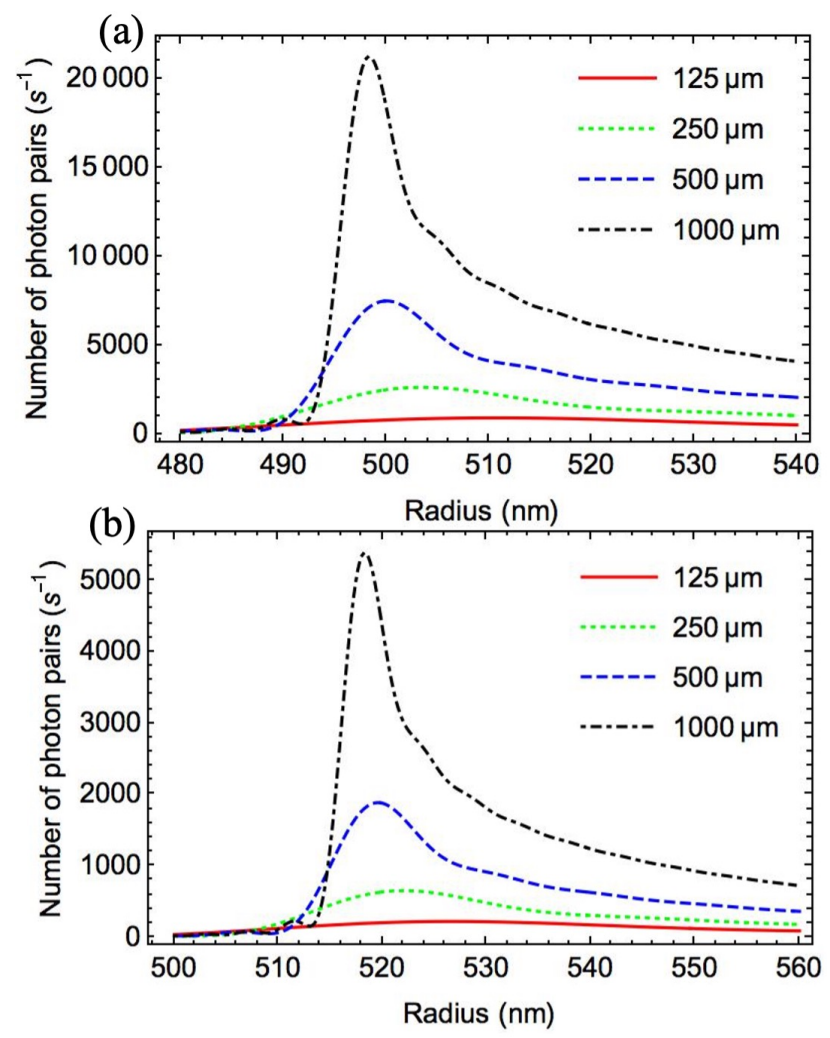

Fig. 8. Number of photon pairs generated per second in a silica nanofiber of different lengths and for a $1 \mathrm{~W}$ input power in a silica-PTFE architecture. (a) $T M_{01}$ input mode. (b) $H E_{21}$ input mode.

These plots confirm the structure optimization: the peaks are larger, without significant changes in the photon rates.

\subsection{Optimization of surface nonlinearity}

The photon pair rates, even with the optimized architecture described above, remain quite moderate. It is worth noting, that about $75 \%$ of the contribution to coefficient $\rho^{S F G}$ arises from the $\chi_{r r r}^{(2 s)}$ susceptibility coefficient. Enhancing the photon pair rate by reinforcing the $\chi_{r r r}^{(2 s)}$ susceptibility coefficient is thus tempting. One way for achieving this enhancement was proposed and demonstrated in the literature: bilayers of radially self-aligned nonlinear molecules were deposited onto a nanofiber and led to a large increase of the SHG signal compared to the bare fiber [50,51]. Due to the molecule alignment, and conversely to bulk silica, the dipolar second-order bulk susceptibility coefficient differs from zero. It was measured equal to $\chi_{r r r}^{(B u l k)}=21 \mathrm{pm} . \mathrm{V}^{-1}$. A bilayer is $1 \mathrm{~nm}$ thick and the deposit of up to 150 bilayers was demonstrated [52]. Because the total thickness $h$ of these bilayers is much smaller than both the optical wavelength and the nanofiber diameter, we can still treat such a deposit as a surface nonlinearity by setting the equivalence

$$
\chi_{r r r}^{(2 s)}=h \chi_{r r r}^{(B u l k)},
$$


For instance, with 10 bilayers, $h=10 \mathrm{~nm}$, we obtain for such an arrangement $\chi_{r r r}^{(2 s)}=$ $21010^{3} \mathrm{pm}^{2} \cdot \mathrm{V}^{-1}$. That is more than a 30 -fold improvement compared to the bare silica architecture. Because the photon pair rate scales as the square of the nonlinear susceptibility, the presence of the highly nonlinear functional bilayers increases the photon pair rate by a factor of about 1000 compared to a bare silica nanofiber.

\section{Conclusion}

We have presented a design of an all-fiber source of correlated photon pairs based on standard telecommunication tapered fibers. The involved second-order parametric fluorescence process relies on the surface dipole and bulk multipole nonlinearities.

We illustrated our vectorial modelling by considering the case of a pump beam at wavelength $775 \mathrm{~nm}$ in one of the two lowest order nanofiber modes $T M_{01}$ or $H E_{21}$, the pairs of photons are created around $1550 \mathrm{~nm}$ in the vicinity of degeneracy in the fundamental $H E_{11}$ nanofiber mode. For the $T M_{01}$ pump beam case, the two correlated photons are always produced with the same polarization, either $H E_{11}^{\text {even }}$ or $H E_{11}^{\text {odd }}$. For the $H E_{21}$ pump mode case, we demonstrated that a given orientation of the output polarizers leads to correlated photons bearing the same polarization, while another choice produces orthogonally polarized photons.

We demonstrated that such sources, built around currently available naked silica nanofibers, could generate up to $N_{p h} \approx 22000$ pairs/s in the $H E_{11}$ modes with a $1 \mathrm{~W}$ pump power in a $T M_{01}$ mode.

We suggested two possibilities to considerably increase these generation rates: embedding the nanofiber in a medium with a carefully selected dispersion for noncritical modal phase matching; depositing a thin layer of a highly nonlinear functional molecules between the silica nanofiber and embedding medium.

Such optimized structures should increase the generation rates by several orders of magnitude.

Disclosures. The authors declare that there are no conflicts of interest related to this article.

\section{References}

1. A. Orieux, and E. Diamanti, "Recent advances on integrated quantum communications," J. Opt. 18, 083002 (2016).

2. F. Flamini, N. Spagnolo, and F. Sciarrino, "Photonic quantum information processing: a review," Rep. Prog. Phys. 82, 016001 (2019).

3. S. Slussarenko, and G. J. Pryde, "Photonic quantum information processing: A concise review," Appl. Phys. Rev. 6, 041303 (2019).

4. L. M. Duan, and C. Monroe, "Colloquium: Quantum networks with trapped ions," Rev. Mod. Phys. 82, 1209 (2010).

5. J. L. O'Brien, A. Furusawa, and J. Vučković, "Photonic quantum technologies," Nature Photon. 3, 687-695 (2009).

6. X. Li, P. L. Voss, J. E. Sharping, and P. Kumar, "Optical-Fiber Source of Polarization-Entangled Photons in the $1550 \mathrm{~nm}$ Telecom Band," Phys. Rev. Lett. 94, 053601 (2005).

7. H. Takesue, and K. Inoue, "Generation of polarization-entangled photon pairs and violation of Bell's inequality using spontaneous four-wave mixing in a fiber loop," Phys. Rev. A 70, 031802 (2004).

8. J. Fulconis, O. Alibart, J. L. O'Brien, W. J. Wadsworth, and J. G. Rarity, "Nonclassical Interference and Entanglement Generation Using a Photonic Crystal Fiber Pair Photon Source,” Phys. Rev. Lett. 99, 120501 (2007).

9. L. Cui, X. Li, C. Guo, Y. H. Li, Z. Y. Xu, L. J. Wang, and W. Fang, "Generation of correlated photon pairs in micro/nano-fibers," Opt. Lett. 38, 5063-5066 (2013).

10. H. Pourbeyram and A. Mafi, "Photon pair generation with tailored frequency correlations in graded-index multimode fibers," Opt. Lett. 43, 2018-2021 (2018).

11. K. Rottwitt, J. G. Koefoed, and E. N. Christensen, "Photon-pair sources based on intermodal four-wave mixing in few-mode fibers," Fibers 6, 32 (2018).

12. S. Dong, L. Yu, W. Zhang, J. Wu, W. Zhang, L. You and Y. Huang, "Generation of hyper-entanglement in polarization/energy-time and discrete-frequency/energy-time in optical fibers," Sci. Rep. 5, 9195 (2015).

13. P. G. Kwiat, K. Mattle, H. Weinfurter, A. Zeilinger, A. V. Sergienko, and Y. Shih, "New High-Intensity Source of Polarization-Entangled Photon Pairs,” Phys. Rev. Lett. 75, 4337 (1995). 
14. J. Belhassen, F. Baboux, Q. Yao, M. Amanti, I. Favero, A. Lemaître, W. S. Kolthammer, I. A. Walmsley, and S. Ducci, "On-chip III-V monolithic integration of heralded single photon sources and beamsplitters," Applied Phys. Lett. 112, 071105 (2018).

15. T. Suhara, "Generation of quantum-entangled twin photons by waveguide nonlinear-optic devices," Laser \& Photon. Rev. 3, 370-393 (2009).

16. S Tanzilli, A. Martin, F. Kaiser, M. P. DeMicheli, O. Alibart, and D. B. Ostrowsky, "On the genesis and evolution of integrated quantum optics," Laser Photonics Rev. 6, 115 (2012).

17. E. Y. Zhu, Z. Tang, L. Qian, L. G. Helt, M. Liscidini, J. E. Sipe, C. Corbari, A. Canagasabey, M. Ibsen, and P. G. Kazansky, "Direct Generation of Polarization-Entangled Photon Pairs in a Poled Fiber," Phys. Rev. Lett. 108, 213902 (2012).

18. C. Chen, A. Riazi, E. Y. Zhu, M. Ng, A. V. Gladyshev, P. G. Kazansky and L. Qian, “Turn-key diode-pumped all-fiber broadband polarization-entangled photon source," OSA Continuum. 1, 981 (2018).

19. F. Xu, Z. X. Wu, and Y. Q. Lu, "Nonlinear optics in optical-fiber nanowires and their applications," Prog. Quantum. Electron. 55, 35 (2017).

20. G. Brambilla, “Optical fibre nanowires and microwires: a review,” J. Opt. 12, 043001 (2010).

21. K. P. Nayak, M. Sadgrove, R. Yalla, F. L. Kien, and K. Hakuta, "Nanofiber quantum photonics,” J. Opt. 20, 073001 (2018).

22. M. Cao, H. Li, M. Tang, Y. Mi, L. Huang, and G. Ren, "Forward stimulated Brillouin scattering in optical nanofibers,” J. Opt. Soc. Am. B 36, 2079-2086 (2019).

23. G. Fanjoux, J. Chrétien, A. Godet, K. Phan-Huy, J-C. Beugnot, and T. Sylvestre, "Demonstration of the evanescent Kerr effect in optical nanofibers," Opt. Express 27, 29460-29470 (2019).

24. T. A. Birks and Y. W. Li, “The shape of fiber tapers," J. Lightwave Technol. 10, 432-438 (1992).

25. R. P. Kenny, T. A. Birks, and K. P. Oakley, "Control of optical fibre taper shape,” Electron. Lett. 27, 16541656 (1991).

26. A. W. Snyder, and J. D. Love, "Optical Waveguide Theory,” (New York, NY, USA: Chapman \& Hall, 1983).

27. M. D. Turner, T. M. Monro, and S. Afshar V., "A full vectorial model for pulse propagation in emerging waveguides with subwavelength structures part II: Stimulated Raman Scattering,” Opt. Express 17, 1156511581 (2009)

28. M. Kolesik and J. V. Moloney, "Nonlinear optical pulse propagation simulation: From Maxwell's to unidirectional equations," Phys. Rev. E 70, 036604 (2004).

29. X. Chen, N. C. Panoiu, and R. M. Osgood, "Theory of Raman-Mediated Pulsed Amplification in Silicon-Wire Waveguides," IEEE J. Quantum Electron. 42, 160 (2006).

30. S. Afshar V., and T. M. Monro, "A full vectorial model for pulse propagation in emerging waveguides with subwavelength structures part I: Kerr nonlinearity," Opt. Express 17, 2298-2318 (2009).

31. R. W. Boyd, "Nonlinear Optics," (Academic Pres, San Diego, CA, 1992).

32. N. Bloembergen, R. K. Chang, S. S. Jha, and C. H. Lee, "Optical Second-Harmonic Generation in Reflection from Media with Inversion Symmetry," Phys. Rev. 174, 813 (1968).

33. T. F. Heinz, "Second-order nonlinear optical effects at surfaces and interfaces," in Modern Problems in Condensed Matter Sciences, Elsevier, 29, 353-416 (1991).

34. B. Dick, A. Gierulski, and G. Marowsky, "Determination of the nonlinear optical susceptibility $\chi(2)$ of surface layers by sum and difference frequency generation in reflection and transmission," Appl. Phys. B 38, 107-116 (1985).

35. J. Laegsgaard, “Theory of surface second-harmonic generation in silica nanowires,” J. Opt. Soc. Am. B 27, 1317-1324 (2010).

36. R. S. Bennink, "Optimal collinear Gaussian beams for spontaneous parametric down-conversion," Phys. Rev. A 81, 053805 (2010).

37. A. Ling, A. Lamas-Linares, and C. Kurtsiefer, "Absolute emission rates of spontaneous parametric downconversion into single transverse Gaussian modes," Phys. Rev. A 77, 043834 (2008).

38. J-L. Smirr, M. Deconinck, R. Frey, I. Agha, E. Diamanti, and I. Zaquine, “Optimal photon-pair single-mode coupling in narrow-band spontaneous parametric downconversion with arbitrary pump profile,” J. Opt. Soc. Am. B 30, 288-301 (2013).

39. K. Bencheikh, E. Huntziger, and J. A. Levenson, "Quantum noise reduction in quasi-phase-matched optical parametric amplification,” J. Opt. Soc. Am. B 12, 847-852 (1995).

40. F. Devaux, and E. Lantz, "Spatial and temporal properties of parametric fluorescence around degeneracy in a type I LBO crystal,” Eur. Phy. J. D 8, 117-124 (2000).

41. T. Suhara and H. Kintaka, "Quantum theory analysis of twin-photon beams generated by parametric fluorescence," IEEE J. of Quantum Electron. 41, 1203-1212 (2005).

42. Y. Xu, W. Fang, and L. Tong, "Real-time control of micro/nanofiber waist diameter with ultrahigh accuracy and precision," Opt. Express 25, 10434 (2017).

43. A. Godet, A. Ndao, T. Sylvestre, V. Pecheur, S. Lebrun, G. Pauliat, J-C. Beugnot, and K. P. Huy, "Brillouin spectroscopy of optical microfibers and nanofibers," Optica 4, 1232-1238 (2017).

44. U. Wiedemann, K. Karapetyan, C. Dan, D. Pritzkau, W. Alt, S. Irsen, and D. Meschede, "Measurement of submicrometre diameters of tapered optical fibres using harmonic generation," Opt. Express 18, 7693-7704 (2010). 
45. M. Sumetsky and Y. Dulashko, "Radius variation of optical fibers with angstrom accuracy," Opt. Lett. 35, 23 (2010).

46. A. Azzoune, P. Delaye, and G. Pauliat, "Optical microscopy for measuring tapered fibers beyond the diffraction limit," Opt. Express 27, 24403-24415 (2019).

47. M. Santandrea, M. Stefszky, V. Ansari, and C. Silberhorn, "Fabrication limits of waveguides in nonlinear crystals and their impact on quantum optics applications," New J. Phys. 21, 033038 (2019).

48. E. J. Lim, S. Matsumoto, and M. Fejer, "Noncritical phase matching for guided-wave frequency conversion," Appl. Phys. Lett. 57, 2294-2296 (1990).

49. M. K. Yang, R. H. French, and E. W. Tokarsky, "Optical properties of Teflon®AF amorphous fluoropolymers," J. Micro/Nanolith MEMS MOEMS 7, 033010 (2008).

50. Y. Xua and A. Wang, "Proposal and analysis of a silica fiber with large and thermodynamically stable second order nonlinearity," Appl. Phys. Lett. 90, 211110 (2007).

51. C. Daengngam, M. Hofmann, Z. Liu, A. Wang, J. R. Heflin, and Y. Xu, "Demonstration of a cylindrically symmetric second-order nonlinear fiber with self-assembled organic surface layers," Opt. Express 19, 1032610335 (2011).

52. C. Daengngam, I. Kandas, I. Ashry, J-A Lee, A. Wang, J. R. Heflin, and Y. Xu, "Fabrication and characterization of periodically patterned silica fiber structures for enhanced second-order nonlinearity," Opt. Express 23, 8113-8127 (2015) and “publisher's note” Opt. Express 25, 10473-10473 (2017). 